\title{
An Energy-Efficient Topology Control Algorithm Based on Reinforcement Learning for Wireless Sensor Networks
}

\author{
Thien T. T. Le and Sangman Moh \\ Dept. of Computer Engineering, Chosun University, Gwangju, South Korea \\ thanhthien92003@yahoo.com,smmoh@chosun.com
}

\begin{abstract}
Network connectivity is a key issue in wireless sensor networks (WSNs). Nodes have to establish and maintain a connected topology in a WSN while dealing with interference and packet loss. Topology control techniques allow the network nodes to reduce their transmission power while preserving the network connectivity. In this paper, we present a reinforcement-learning-based communication range control (RL-CRC) algorithm to adaptively adjust the communication range at each sensor node while ensuring the network connectivity in dynamic WSNs. In the proposed $R L-C R C$, the reinforcement learning is exploited to discover neighbors with low interference, and the network topology is effectively obtained in presence of interference. The reinforcement learning based on the so-called Q-learning adapts to changes of node connectivity and, thus, the nodes discover their neighbors and then adaptively control their communication range accordingly. The simulation results show that RL-CRC reduces energy consumption significantly compared to the conventional schemes while maintaining almost the same average communication range and node degree.
\end{abstract}

Keywords: Wireless sensor network, topology control, network connectivity, reinforcement learning, energy efficiency

\section{Introduction}

Wireless sensor networks (WSNs) are broadly used in many application areas such as environmental monitoring, disaster surveillance, traffic control and health care. In WSNs, the process of data gathering normally involves multi-hop communication from sensors to the sink node and, thus, nodes have to discover their neighbors to create a topology which aims to ensure the network coverage and connectivity [1]. The topology control algorithms aim to provide the network coverage to sensor node while maintaining the energy resource of each node. The network coverage refers to the sensors' placement and the cooperation among sensors whereas the network connectivity relates to the message delivery in the network. However, there exist some issues of topology control such as the energy conservation of sensor nodes, limited bandwidth, dynamic network topology, and interference. Because the topology control can affect the connectivity and lifetime of network, it is highly needed to improve energy utilization for prolonging network lifetime and to reduce interference between nodes by considering the network link characteristics.

The node degree in a network means the number of neighbors that one node can communicate directly in a single hop. The $k$-connected network is the network that is still connected after we remove $(k-1)$ nodes. If the node degree is the order of $\Theta(\log n)$, where $n$ is the total number of nodes in a network, the network is connected with high probability [2]. Therefore, many algorithms have been used to achieve the desired node degree in which the network connectivity is obtained with high probability [3-4].

In dynamic WSNs, the machine learning becomes a new solution for optimizing resource utilization and minimizing the energy consumption of sensor nodes [5]. In [6], the theory of reinforcement learning algorithm is introduced to adapt to an unknown 
environment. The new state is predicted on the basis of the present state while interacting with the unknown environment, the probability of changing to a new state, and the reward of changing. Usually, the reinforcement learning uses $Q$-learning method to get the optimal actions. In WSNs, the reinforcement learning algorithm has been applied to medium access control protocols, clustering algorithms, localization and object targeting, and data integrity and fault detection solutions [5]. In addition, the reinforcement learning has been applied to some protocols in WSNs such as routing and rate control [6].

In this paper, we exploit the reinforcement learning to maintain the connected topology with the desired degree, which aims to save the transmission power of nodes. The proposed reinforcement-learning-based communication range control (RL-CRC) algorithm learns the varying network link characteristics using the reinforcement learning technique and gives an optimal choice of node degree. The RL-CRC algorithm calculates the node degree or the number of neighbors and ensures the connectivity and reachability between any two nodes. The less the degree is, the less the number of neighbors is in the node's communication range. Therefore, the number of interfered links can be decreased. According to our performance study, RL-CRC consumes much less energy than the conventional schemes while maintaining almost the same average communication range and node degree with the desired number of neighbors.

The rest of this paper is organized as follows: In the following section, we review the existing works for topology control in WSNs. In Section 3, the RL-CRC algorithm is presented and discussed. In Section 4, the performance of the proposed RL-CRC is evaluated via computer simulation and compared to that of the conventional algorithms. Finally, the paper is concluded in Section 5.

\section{Related Works}

A WSN usually consists of a large number of sensor nodes which communicate to the sink node by one-hop or multi-hop links. In WSNs, some aspects should be taken into design consideration while maintaining the communication links, which include energy conservation, limited bandwidth, unstructured and time-varying network topology, lowquality communications, and scalability. However, two main issues of the topology control in WSNs are network coverage and network connectivity [1]. The taxonomy of network coverage consists of blanket coverage, barrier coverage, and sweep coverage which describe the quality of network coverage. In addition, the network connectivity can be categorized as temporal control and spatial control. The temporal control is considered as power management mechanism by controlling the duration of active mode and sleep mode of sensor nodes. The spatial control mainly aims to adjust the transmission power of sensor nodes. In [8], the topology control algorithms focus on maintaining a connected topology with the minimal energy consumption at nodes. The first task of performing topology control is to guarantee the connectivity of a network by adapting the communication range of each node so that the network is completely connected. As a consequence, the communication range or network connectivity can affect the network topology and the communication quality. However, increasing the communication range of nodes can cause interference to other simultaneous transmissions. Therefore, it is necessary to control the topology in WSNs to reduce the interference and to guarantee the network connectivity.

Some topology control algorithms for wireless ad hoc networks are summarized in [9]. The algorithms use the set of neighbor nodes with their locations to create the topology. However, the neighbor discovery at each node is based on a number of broadcast messages, resulting in more energy dissipation. Recently, machine learning techniques are applied to WSNs to maximize the utilization of network resources [5]. In fact, some algorithms based on the machine learning techniques for topology control have been studied for minimizing the transmission power and communication range by maintaining 
the set of neighbors. In [10], a distributed algorithm is used to calculate the per-node minimum transmission power in the topology control. A new link interference model and a centralized topology control algorithm are presented to reduce link interference over the network in [11]. The algorithm is based on the centralized topology control which updates the interference links according to the interference weight. The communication links between two nodes can be updated or deleted to preserve the energy resource. In [12], the network topology is controlled by using a learning-based method which broadcasts request messages and receives acknowledgement from the receiver. The algorithm consists of message broadcasting steps during which each node discovers its neighbors according to the received messages. At first, nodes use the maximum communication range and then decrease the communication range by half. This algorithm is simple and needs only the received messages of neighbors, but it does not guarantee the energy efficiency. In [13], a self-organized backbone node capable of topology control (STABWIN) aims to reconstruct the backbone and topology in wireless networks. The operation of STAB-WIN consists of three steps as follows: The first step is backbone formation which creates a connected dominated set. The second step is called backbone reconstruction, and topology control aims to identify the one hop nodes for alternate routing. The last step is self-organization to build a scalable network.

In [14], the reinforcement learning technique is applied to discover the varying network link. A node discovers the network and then selects its neighbors based on the desired node degree while preserving the network connectivity. Each node considers the packet delivery and the energy usage in choosing its neighbors. Another reinforcement learning technique is used to establish the connected topology in the presence of interference [15]. This method focuses on multi-channel switching to avoid interference, and each node is capable to switch the working channel and to connect to another node. In [16], a graphbased topology construction algorithm is used to formulate the communication topology for WSNs. In the fuzzy logic topology control (FTC) scheme [3], it is shown that the node degree in dynamic WSNs can be maintained to achieve high connectivity with low communication range. The fuzzy logic controller is used in each node to adapt the network connectivity without the information of neighbors. Each node has a training data set for the design of network construction. The local minimum spanning tree (LTRT) scheme [4] also maintains the network degree by applying the spanning tree construction algorithm $k$ times.

However, some topology control algorithms are mainly localized, in which it is difficult to adapt to the time-varying network topology. Most of the algorithms use the information of neighbors to create a topology. Unlike the other works that focus on the number of interference links in the communication range, our work in this paper focuses on minimizing the communication range. Our work also tries to maintain the node degree by using the reinforcement learning algorithm, rather than using a training data set as in [3]. By exploiting the reinforcement learning, nodes can learn the environment and, thus, discover their neighbors and adjust their communication range adaptively. Therefore, our proposed RL-CRC algorithm is energy-efficient and adaptive to dynamic networks.

\section{Communication Range Control}

In this section, our topology control algorithm based on reinforcement learning, which is named RL-CRC as introduced in Section 3.1, is presented and discussed in detail.

\subsection{Network Model}

A WSN consists of many sensor nodes and a sink node where the sensor nodes send the sensed data to sink node. The sensor nodes have a certain limitation such as energy, storage, and radio communication capabilities. A WSN can be modeled as an undirected network $G(V, E)$ with a set of vertices $V$ as its nodes and a set of edges $E$ as links between 
nodes. In this paper, a reinforcement learning algorithm is designed to achieve the desired node degree. The algorithm aims to check for strong connectivity and to select the optimal topology. This algorithm runs at each sensor node which collects information from its neighbors and adjusts the communication range to achieve the desired node degree. Because this algorithm requires only the information of one-hop neighbors, it can be effectively used in large-scale WSNs while saving energy.

The system model for topology control consists of two main phases: topology construction and topology learning. Given a network $n$ nodes, the network topology is controlled by an algorithm at each node to ensure the network connectivity. In the first phase, the system will generate a random topology. Each node broadcasts the HELLO message with the maximum communication range $R_{\max }$ and, then, it collects the information of its neighbors. In the second phase, the sensor nodes run the reinforcement learning algorithm to construct an optimal topology with low communication range and high connectivity. In this paper, we only consider undirected links, and node $x$ is the neighbor of node $y$ if node $x$ is in the communication range of node $y$. However, the communication range of node $y$ can be updated after sometime and, then, node $x$ may not be a neighbor of node $y$. Therefore, the reinforcement algorithm is used to update the neighbors of nodes $x$ and $y$ while their communication ranges are changed.

\subsection{Reinforcement Learning for Communication Range Control}

In our proposed RL-CRC, the network connectivity in a WSN is established as follows: The agents of the reinforcement learning (RL) are the nodes which carry out the task to establish the network connectivity. The state of environment $(S)$ of each agent (node $i$ ) is given by set $N_{i}$ of its neighbors so that $S=\left\{k_{1}, k_{2}, \ldots, k_{\max }\right\}$, where $k_{\max }$ is the maximum desired node degree. At each state $s \in S$, a node performs an action $(a)$ which changes the communication range of the node. The agent performs an action that interacts with the environment and, then, gets the reward $(R W)$ as shown in Figure 1. Hence, the agent will decide to take an action based on this reward to reach the desired state. In this paper, the desired state or the objective of RL is to ensure the $k$-connected network where the node degree of each node is $\mathrm{k}$.

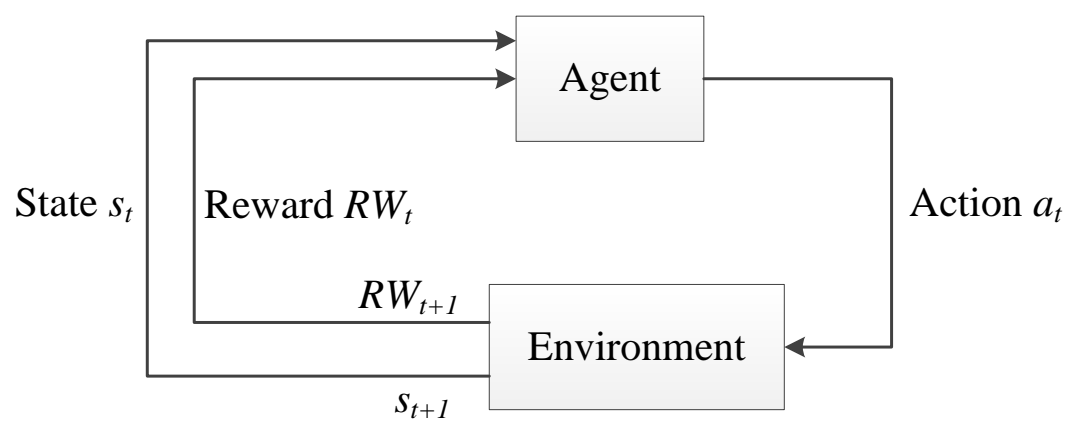

Figure 1. Interaction between the Agent and the Environment in RL

Reward Function A reward function is used by RL agents to calculate the immediate reward of taking an action. Both the network coverage and the network connectivity are critical for topology control. Because the node degree or the number of neighbors of a node is highly correlated with the communication range of the node, we choose to employ the node's degree $k$ as the state $s$ and the communication range $r$ represents the action $a$ generated.

To evaluate the effective communication range and the number of neighbors at time $t$, we denote the number of neighbors of node $i$ as the node's degree $k_{t, i}$ with the 
communication range $r_{t, i}$. The reward function of node $i, R W_{t, i}$, comprises of two independent components as follows

$$
R W_{t, i}=1-\omega_{k} \frac{k_{t, i}}{K_{\max }}-\omega_{r} \frac{r_{t, i}}{R_{\max }}
$$

where $K_{\max }$ is the desired node degree, $R_{\max }$ is the maximum communication range of node $i$, and $\omega_{k}$ and $\omega_{r}$ are weighting coefficients of the node degree and the communication range, respectively.

Q-Learning Algorithm In our reinforcement learning, the RL agent evaluates the temporal difference, updates the $Q$-value and selects the next action according to the $\varepsilon$ greedy method [7]. The action space $A(s)$ for a given state $s$ is specified to be a subset of A. In the communication range control strategy based on the Q-learning, a node is assumed to use the $\varepsilon$-greedy method to choose its communication range, where the communication range with the maximum $Q$-value taken in the state $s_{t}$ is chosen with a high probability $\left(1-\varepsilon_{t}\right)$ while other communication ranges are taken with an equal low probability. $Q$-learning methods applies the $\varepsilon$-greedy action selection, where $\varepsilon=0.1$ as in [7]. By considering the maximum $Q$-value at state $s$ for all the possible actions $A(s)$, the probability $\pi(s, a)$ to choose action $a$ is represented as

$$
\pi(s, a)= \begin{cases}1-\frac{\varepsilon}{|A(s)|} & \text { if } a=a^{*} \\ \frac{\varepsilon}{|A(s)|} & \text { otherwise }\end{cases}
$$

$$
\begin{gathered}
a^{*}=\max _{A(s)} Q(s, a) \\
\text { where Then, the } Q \text {-value for state } s \text { and action } a \text { is defined as } \\
Q(s, a)=R(s, a)+\gamma \max _{A(s)} Q\left(s^{\prime}, a\right)
\end{gathered}
$$

where $\gamma$ is the learning constant in order to adapt to the non-stationary environment. In this algorithm, we use learning constant $\gamma=0.5$. The $Q$-learning is calculated by the summation of the expected reward at state $s$ with action $a$ and the highest $Q$-value for the next state with all possible actions in subset $A(s)$. The $Q$-learning algorithm for communication range control is given in Figure 2.

Topology Control at a Sensor Node Each node broadcast the HELLO message with the maximum communication range $R_{\max }$ and, then, it collects the information of its neighbors. According to the received information, each node runs the RL algorithm to modify its communication range to achieve the desired node degree. The proposed RLCRC algorithm for topology control is shown in detail in Figure 3. This algorithm runs at each node in a WSN. After receiving the messages from the neighbors, each node chooses an initial state $s$ which is equal to the number of neighbors of the node as in lines 4-5. From lines 7 to 9 , the node chooses an action $a_{\max }$ and then runs the $Q$-learning algorithm as in Figure 2. The chosen communication range of the node is $a_{\max }$ at state $s$. 
1. Initialize $A(s)$ for all $s \in S$; Initialize $\gamma, \varepsilon$;

2. Set $Q(s, a)=0 \forall s \in S, \forall a \in A(s)$;

3. Loop for $t=1,2$..

4. While $\left(s_{k} \sim=K_{\max }\right)$

5. Choose current state $s_{k}=k_{t}$;

6. For $r_{r}$ in 0 to $R_{\max }$

7. Choose action $a_{k}=r_{r}$ using the policy derived from $Q$ ( $\varepsilon$-greedy) as in (2)

8. $\quad$ End For

9. Take action $a_{k}=r_{r}$, and observe reward $r\left(s_{k}, a_{k}\right)$ and next state $s_{t+1}\left(k_{t+1}, r_{r}\right)$

10. Update Q: $Q\left(s_{t}, r_{t}\right)=r\left(s_{k}, a_{k}\right)-\gamma * \max Q_{t}\left(s_{t+1}, r_{t}\right)$

11. Update next state $s_{t+1}$ to current state

12. End While

13. End Loop

Figure 2. Q-Learning Algorithm for Communication Range Control

Initialize: $R_{\max }$, rounds

1. $r_{t} \leftarrow R_{\max }$

2. Broadcast HELLO message with current transmission power and communication range

3. Receive the messages from the others

4. Calculate the number of current neighbors

5. Choose an initial state $s$

6. While rounds $>0$

7. Choose $a_{\max }$ for $s$

8. Update communication range by $Q$-learning algorithm

9. rounds $=$ rounds -1

10. End while

Figure 3. Algorithm for Topology Control at each Sensor Node

\section{Performance Evaluation}

In this section, the proposed RL-CRC algorithm is evaluated and compared to the existing works by using Matlab. That is, the proposed algorithm is compared with the conventional algorithms of FTC [3] and LTRT [4] as well as the case without topology control. The FTC algorithm [3] uses fuzzy logic control and a training data set to maintain the desired node degree of around 3. The LTRT algorithm [4] performs the spanning tree construction algorithm multiple times to construct the topology which achieves low degree and low communication range. 


\subsection{Simulation Environment}

In our simulation, nodes are uniformly deployed at random in a $100 \times 100 \mathrm{~m}^{2}$ field as in [3]. The communication ranges vary from 0 to $30 \mathrm{~m}$ (which is the maximum communication range $R_{\max }$ in our simulation). Each node is capable to adjust the communication range within $\left[0, R_{\max }\right]$. As in [3] and [4], a network is fully connected when the node degree is 4 . Therefore, we set the desired degree of nodes as $K_{\max }=4$. In order to simulate different node densities, the number of nodes deployed in the area varies from 50 to 90 nodes. In the Q-learning algorithm, we choose the learning constant $\gamma=0.5$, and the weighting coefficients $\omega_{k}$ and $\omega_{r}$ in reward functions are 0.5 and 0.5 , respectively. The simulation has been iterated 40 times for each scenario. The number of rounds for RL-CRC is chosen as 10 to achieve the desired node degree. The simulation parameters are summarized in Table 1.

Table 1. Simulation Parameters

\begin{tabular}{|c|c|}
\hline Parameter & Value \\
\hline Number of nodes & $50 \sim 90$ \\
\hline Maximum communication range & $30 \mathrm{~m}$ \\
\hline Network area & $100 \times 100 \mathrm{~m}^{2}$ \\
\hline Desired node degree & 4 \\
\hline Learning constant & 0.5 \\
\hline Weighting coefficient of the node degree $\left(\omega_{k}\right)$ & 0.5 \\
\hline Weighting coefficient of the communication range $\left(\omega_{r}\right)$ & 0.5 \\
\hline Number of rounds & 10 \\
\hline
\end{tabular}

\subsection{Simulation Results and Discussion}

Figure 4 shows the examples of the resulting topology when both no topology control and the proposed RL-CRC are applied to the network of 50 nodes with $k=4$. With RL$\mathrm{CRC}$, the network is fully covered and every node connects to the nearest nodes. It is clearly shown in the figure that the communication ranges of nodes with RL-CRC are reduced significantly compared to those with no topology control.

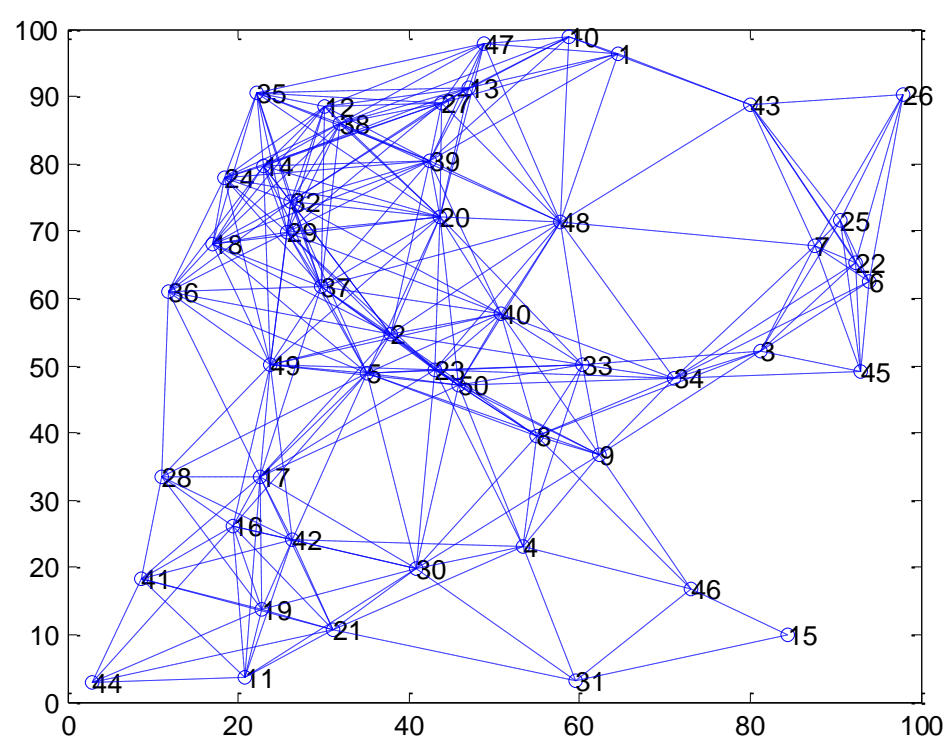

(a) No Topology Control 


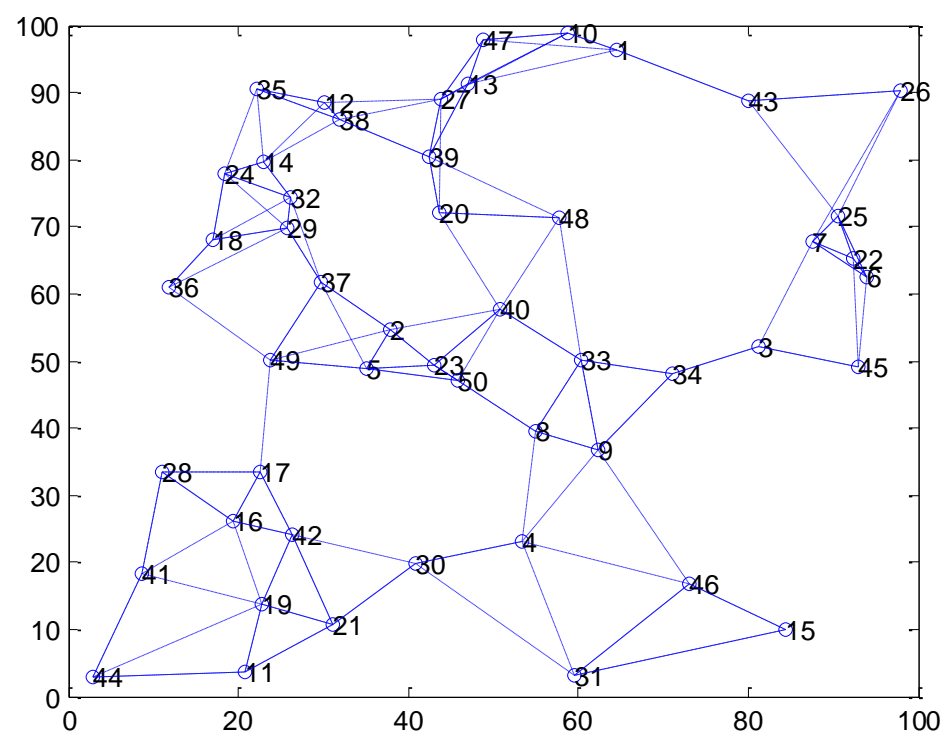

(b) RL-CRC

Figure 4. Examples of the Resulting Topology $(n=50, k=4)$

In [17], energy expended ratio (EER), which is the ratio of the average power of a node over the maximum power of a node in a network, is used for a metric of energy efficiency. For simplicity of comparison, in this paper, EER is calculated as the ratio of average communication range to the maximum communication range, and it should be as small as possible. Figure 5 shows EER for different topology control schemes. RL-CRC achieves the lowest EER compared to FTC and LTRT as well as no topology control. That is, the proposed RL-CRC algorithm reduces energy consumption significantly compared to the conventional schemes. This is mainly due to the relatively short average communication range of RL-CRC.

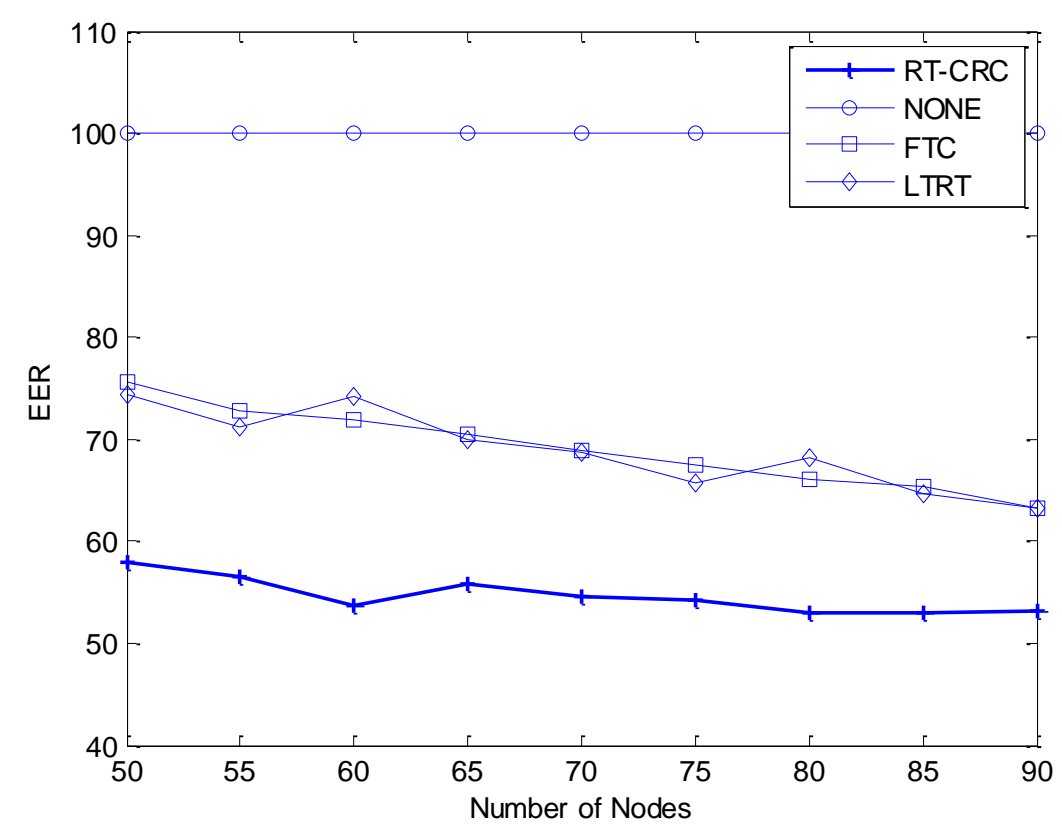

Figure 5. Energy Expended Ratio 
Figure 6 shows that the node degree is ensured at $K_{\max }$ even when the number of nodes increases from 50 to 90 . As a result, RL-CRC can achieve the desired node degree in any type of node deployment in the network. As expected, in comparison to no topology control (denoted 'NONE' in the graphs), the network with topology control algorithms can reduce the node degree remarkably as shown in the figure. Thanks to the $Q$-learning algorithm at each node, the node controls its communication range to adapt the desired number of neighbors, and the average node degree of RL-CRC is nearly close to that of FTC but it is better than that of LTRT.

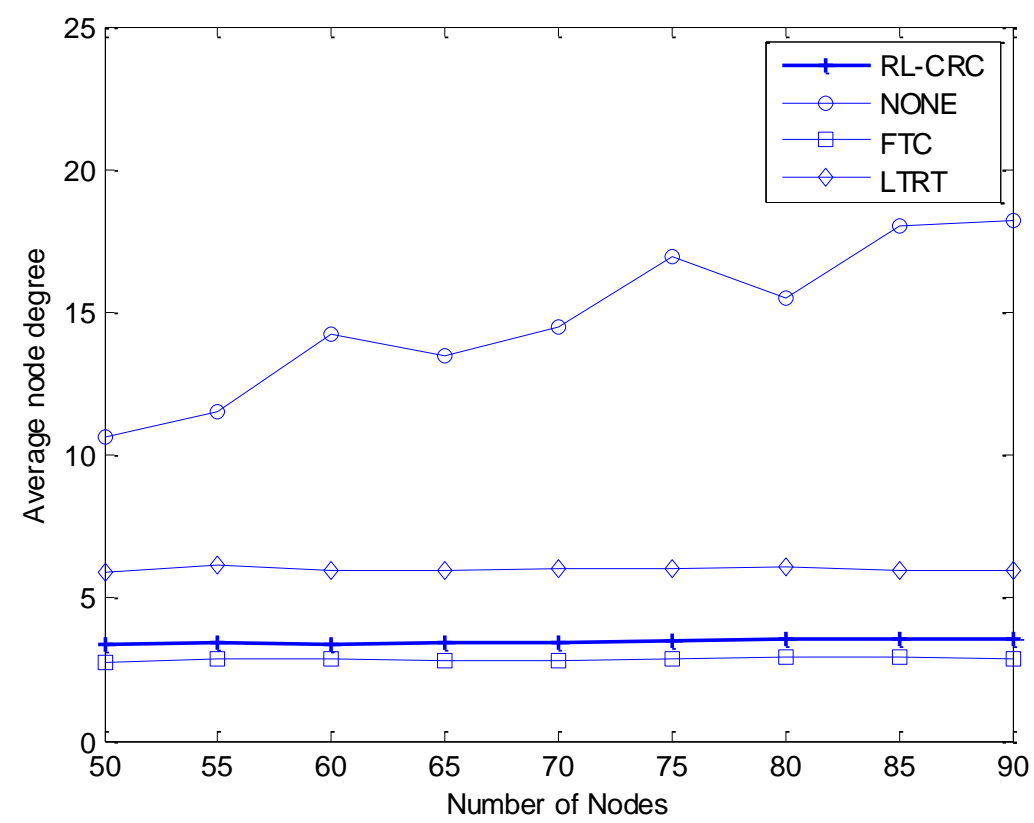

Figure 6. Average Node Degree $(K=4)$

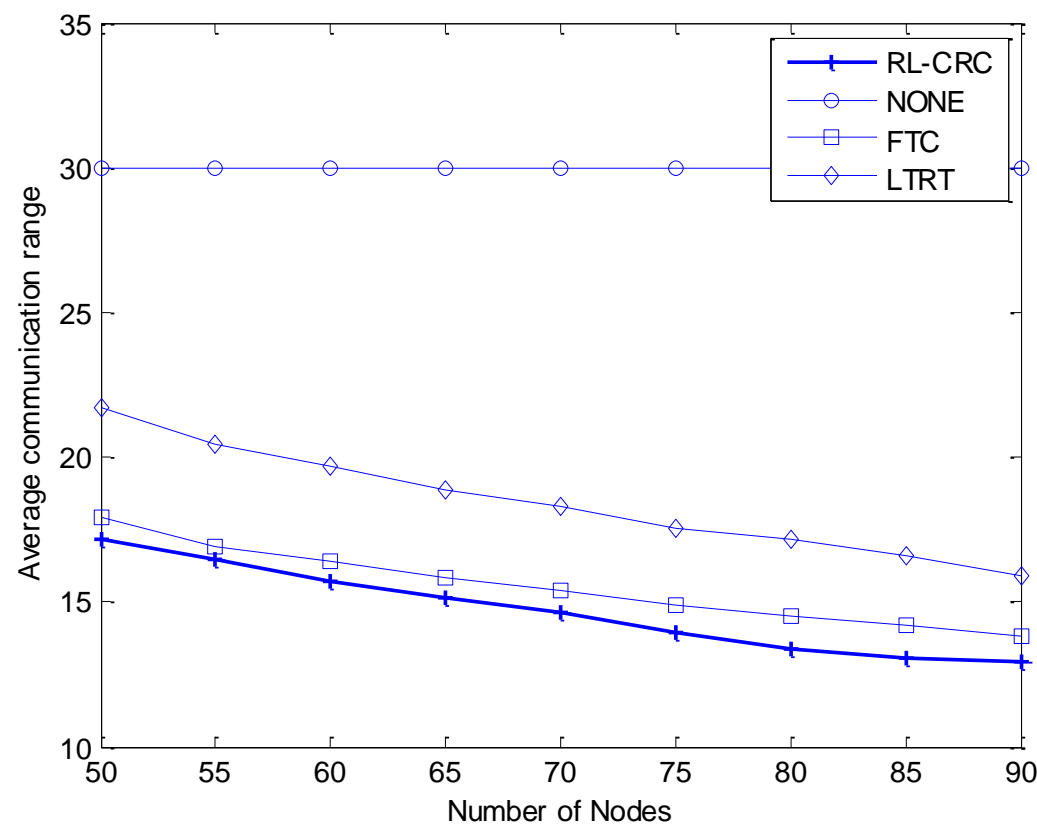

Figure 7. Average Communication Range 
As shown in Figure 7, the average communication range is expected to decrease as the number of nodes increases. The network without topology control needs to communicate with the maximum communication range while that with topology control can reduce the communication range as shown in Figure 7. It is also shown in the figure that the average communication range with topology control decreases when the number of nodes increases due to the dense deployment of nodes. Compared to FTC and LTRT, RL-CRC can achieve the shortest average communication range independent of the number of nodes.

In summary, it is easily inferred from the three performance plots that the proposed RL-CRC reduces energy consumption remarkably compared to the conventional schemes while maintaining almost the same average communication range and node degree.

\section{Conclusion}

In this paper, we have proposed a reinforcement-learning-based communication range control algorithm to energy-efficiently solve the connectivity problem in WSNs. The proposed RL-CRC algorithm controls the communication range of sensor nodes with less energy consumption, improving the network connectivity significantly. RL-CRC can achieve the desired node degree and shorten the average communication range in a WSN, which reduces the wasted energy while ensuring the network connectivity. As a future work, we are going to exploit the mobility of nodes and devise a more resilient topology control protocol without degrading performance.

\section{Acknowledgments}

This paper is a revised and expanded version of a paper entitled "ReinforcementLearning-Based Topology Control for Wireless Sensor Networks" presented at the 3rd International Conference on Green and Smart Technology, Jeju, Korea, Dec. 21-23, 2016 [18]. This research was supported in part by Basic Science Research Program through the National Research Foundation of Korea (NRF) funded by the Ministry of Education (NRF-2016R1D1A1A09918974). Correspondence should be addressed to Dr. Sangman Moh (smmoh@chosun.ac.kr).

\section{References}

[1] M. Li, Z. Li, A. V. Vasilakos, A Survey on Topology Control in Wireless Sensor Networks: Taxonomy, Comparative Study, and Open Issues, Proceedings of the IEEE, vol. 101, no. 12, (2013), pp. 253-2557.

[2] P. Balister, B. Bollobás, A. Sarkar, Percolation, Connectivity, Coverage and Colouring of Random Geometric Graphs. Bollobás, B., Kozma, R., Miklós, D. (eds.) Handbook of Large-scale Random Networks, Springer, Heidelberg, vol. 18, (2006), pp 117-142.

[3] Y. Huang, J-F. Martínez, V.H. Díaz, J. Sendra, A Novel Topology Control Approach to Maintain the Node Degree in Dynamic Wireless Sensor Networks, Sensors, vol.14, no. 3, (2014), pp. 4672-4688.

[4] K. Miyao, H. Nakayama, N. Ansari, N. Kato, LTRT: An Efficient and Reliable Topology Control Algorithm for Ad-hoc Networks, IEEE Transactions on Wireless Communications, vol. 8, no.12, (2009), pp. 6050-6058.

[5] M. A. Alsheikh, S. Lin, D. Niyato, H-P.Tan, Machine Learning in Wireless Sensor Networks: Algorithms, Strategies, and Applications, IEEE Communications Surveys \& Tutorials, vol. 16, no.4, (2014), pp. 1996-2018.

[6] S. Russell, P.Norvig, Artificial Intelligent: A mordern approach, third edition. Prentice Hall, (2003).

[7] K.-L.A.Yau, H.G. Goh, D. Chieng, K.H. Kwong, Application of Reinforcement Learning to Wireless Sensor Networks: Models and Algorithms, Computing, vol. 97, no.11, (2015), pp.1045-1075.

[8] P. Gober, A. Ziviani, P. Todorova, M. D. de Amorim, P. Hünerberg, S. Fdida, Topology Control and Localization in Wireless Ad-hoc and Sensor Networks, Ad Hoc \& Sensor Networks, vol. 1, no. 4, (2005), pp. 301-322.

[9] Y. Manolopoulos, D. Katsaros, A. Papadimitriou, Topology Control Algorithms for Wireless Sensor Networks: A Critical Survey. Proceedings of the 11th International Conference on Computer Systems and Technologies and Workshop for PhD Students in Computing on International Conference on Computer Systems and Technologies, Sofia, Bulgaria), (2010), pp. 1-10. 
[10] J. Liu, B. Li, Distributed Topology Control in Wireless Sensor Networks with Asymmetric Links. Proceedings of IEEE Global Telecommunications Conference (GLOBECOM 2003), pp. 1257-1262, San Francisco, USA, (2003), Dec. 1-5.

[11] G. Sun, L. Zhao, Z. Chen, G. Qiao, Effective Link Interference Model in Topology Control of Wireless Ad hoc and Sensor Networks, Journal of Network and Computer Applications, (2015), Vol. 52, pp. 6978.

[12] S.H. Khasteh, S.B. Shouraki, A Learning Based Method for Topology Control in Wireless Sensor Networks, Proceedings of 5the International Conference on Autonomic and Autonomous Systems (ICAS 2009), pp. 51-55, Valencia, Spain, (2009), April. 20-25.

[13] S. Smys, G.J. Bala, STAB-WIN: Self Organized, Topology Control Ability Backbone Node in Wireless Networks, Wireless Personal Communications, (2012), Vol. 63, no. 3, pp. 529-548.

[14] J. Shanavas, S. Simi, An Energy Efficient Topology Control Scheme with Connectivity Learning in Wireless Networks. Proceedings of International Conference on Advances in Computing, Communications and Informatics (ICACCI 2014), pp. 1770-1774, Delhi, India, (2014), Sept. 24-27.

[15] L. Stabellini, J. Zander, Interference Aware Self-organization for Wireless Sensor Networks: A Reinforcement Learning Approach. Proceedings of IEEE International Conference on Automation Science and Engineering (CASE 2008), (2008); VA, USA.

[16] D. Kim, E. Noel, K.W. Tang, "WSN Communication Topology Construction with Collision Avoidance and Energy Saving", Proc. of 11the IEEE Consumer Communications and Networking Confference (CCNC), (2014); Las Vegas, US.

[17] M. Hajiaghayi, N. Immorlica, V.S. Mirrokni, "Power Optimization in Fault-tolerant Topology Control Algorithms for Wireless Multi-hop Networks”, IEEE/ACM Transactions on Networking, vol. 15, no.6, (2007), pp. 1345-1358.

[18] Thien T. T. Le and Sangman Moh, "Reinforcement-Learning-Based Topology Control for Wireless Sensor Networks," Proc. of 3rd Int. Conf. on Green and Smart Technology (GST 2016), (2016); Jeju, Korea.
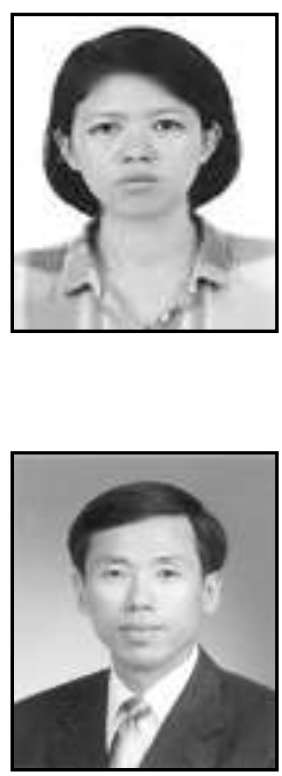

\section{Authors}

Thien T. T. Le, received the B.S. degree in electronics and Telecommunication engineering from Post and Telecommuni-cation Institute of Technology, Viet Nam in 2007 and the M.S. degree in electrical engineering from Ho Chi Minh City University of Technology, Viet Nam in 2011. Currently, she is a Ph.D. student member of Mobile Computing Laboratory, Chosun University, South Korea. Her current research interests include wireless sensor networks and wireless body area networks with a focus on network architectures and protocols.

Sangman Moh, received the Ph.D. degree in computer engineering from Korea Advanced Institute of Science and Technology (KAIST), Korea in 2002. Since late 2002, he has been a professor in the Dept. of Computer Engineering at Chosun University, Korea. From 2006 to 2007, he was on leave at Cleveland State University, USA. Until 2002, he had been with Electronics and Telecommunications Research Institute (ETRI), Korea, where he served as a project leader, since he received the M.S. degree in computer science from Yonsei University, Korea in 1991. His research interests include mobile computing and networking, ad hoc and sensor networks, cognitive radio networks, and parallel and distributed computing systems. He has published more than 200 papers in international and domestic journals and conference proceedings, and has held more than 40 overseas and domestic patents. He serves on the program committees of international conferences and workshops in his areas of interest. Dr. Moh is a member of the IEEE, the ACM, the IEICE, the KIISE, the IEIE, the KIPS, the KICS, the KMMS, the IEMEK, the KISM, and the KPEA. 
International Journal of Control and Automation

Vol. 10, No. 5 (2017) 